\title{
Los Estudios Universitarios y el Mindfulness. Una Revisión Sistemática
}

\author{
The University Studies and the Mindfulness. \\ A Systematic Review
}

\author{
Virginia Viciana ${ }^{1 *}$ \\ Andrés B. Fernández Revelles ${ }^{1}$ \\ Marta Linares ${ }^{1}$ \\ Tamara Espejo ${ }^{2}$ \\ Pilar Puertas ${ }^{1}$ \\ Ramón Chacón ${ }^{3}$ \\ ${ }^{1}$ Universidad de Granada, España \\ ${ }^{2}$ Universidad de Cádiz, España \\ ${ }^{3}$ Universidad de Huelva, España
}

\begin{abstract}
El proceso de formación del estudiante universitario está compuesto por la transmisión de contenidos académicos utilizando para ello estrategias metodológicas de diferente índole. Para que se desarrolle se deberán plantear el desarrollo de competencias emocionales, ayudándole a reconocer tanto sus emociones y motivaciones como las de los demás, facilitando la capacidad de comunicar y escuchar, adaptándose al medio para reaccionar de forma adecuada ante las diversas situaciones. Los programas de mindfulness pretenden reducir el cansancio emocional, fomentar el aprendizaje de técnicas efectivas del manejo del estrés y habilidades de regulación emocional, que ayudan a que se produzca un correcto proceso de enseñanza y aprendizaje. El presente trabajo analiza la evolución de la producción científica que hay en la Web of Science durante los últimos diez años, clasificando éstos según el objetivo de mejora buscado, el número de trabajos según el país de procedencia y los instrumentos de media utilizados, que abordan el mindfulness como un factor influyente en el desarrollo del estudiante universitario, constatando la necesidad de aumentar las investigaciones en este emergente cuerpo de estudio.
\end{abstract}

Descriptores: Estudiante; Universidad; Desarrollo de la carrera; Rendimiento escolar; Escuela.

The process of training the university student is composed of the transmission of academic content using methodological strategies of different kinds. To get it, it must raise the development of emotional skills, helping them to recognize both their emotions and motivations as those of others, facilitating the ability to communicate and listen, adapting to the environment in order to react appropriately to different situations. Mindfulness programs aim to reduce emotional fatigue, promoting the learning of effective techniques of stress management and emotional regulation skills, factors necessary for a proper teaching and learning process to occur. Our research analyzes the evolution of scientific production in the Web of Science during the last ten years, classifying them according to the objective of improvement sought, the number of works according to the country of origin and the media instruments used, which address mindfulness as an influential factor in the development of the university student, confirming the need to increase research in this emerging body of study.

Keywords: Students; University; Career development; Academic achievement; Schools.

*Contacto: vviciana@ugr.es

ISSN: $1696-4713$

www.rinace.net/reice/

revistas.uam.es/reice
Recibido: $\quad 15$ de septiembre 2017

$1^{\text {a }}$ Evaluación: 3 de noviembre 2017

$2^{\text {a }}$ Evaluación: 7 de diciembre 2017

Aceptado: $\quad 22$ de diciembre 2017 


\section{Revisión de la literatura}

La educación universitaria tiene como principal objetivo reforzar las habilidades intelectuales de los estudiantes, así como prepararlos para una etapa como adultos productiva y exitosa. Sin embargo, la realidad del estudiante universitario está cargada de altas tasas de preocupaciones, problemas de sueño, dificultades relacionadas con el estrés y altos niveles de angustia psicológica entre otros (González, 2008; Lindsay et al., 2015), afectando al éxito académico de los estudiantes. Garcés de los Fayos (1995) afirma que la sobrecarga laboral es equiparable a la sobrecarga académica, ya que los estresores del mundo laboral también están presentes en el contexto académico. La investigación ha constatado la presencia del síndrome de burnout en estudiantes universitarios (Caballero, Abello y Palacio, 2007). En los universitarios el burnout se manifiesta a través del cansancio emocional, influyendo de manera significativa en las expectativas de los alumnos para finalizar sus estudios de forma satisfactoria (Martínez et al., 2002). Este síndrome se caracteriza como una respuesta de evitación ante los sentimientos, pensamientos y emociones que producen malestar y sufrimiento. A este aspecto se le une la presencia de hábitos y estilos de vida insalubres en la mayoría de las poblaciones estudiantiles universitarias, provocando problemas de salud mental (Moses, Bradley y O'Callaghan, 2016). El actual panorama educativo requiere que la implicación de los diferentes agentes educativos vaya más allá de la mera transmisión de contenidos académicos (Pegalajar y López Hernáez, 2015).

Estudios recientes (Dvorakova et al., 2017; Sheikhzadeh y Khatami, 2017a) han identificado varios factores que pueden influir en el nivel de angustia psicológica de los estudiantes, como son las dificultades para hacer frente a la carga académica, el deseo de tener éxito académico, así como la obtención de un bajo rendimiento académico (Stallman, 2008). Entre los principales estresores contextuales que los estudiantes universitarios identifican relacionados con el mundo académico según González y Landero (2007) se encuentran la preocupación por el rendimiento académico, la adaptación al ambiente y a las exigencias del mundo universitario, y las preocupaciones en torno a su futuro.

El bienestar psicológico del alumno universitario es motivo de investigaciones actuales. Ryan y Deci (2001) sostienen que hay pocas investigaciones sobre el bienestar psicológico y argumentan que el bienestar psicológico es más que la ausencia de angustia psicológica. Este aspecto confirma la necesidad de investigar también aspectos del funcionamiento positivo con el fin de obtener una mejor compresión de la experiencia de los universitarios. Por tanto, hay una necesidad de intervenciones que promuevan mejorar las habilidades de afrontamiento en los estudiantes para prevenir la angustia y el burnout futuro (De Vibe et al., 2013). En este sentido, Paredes y Sanabria-Ferrand (2008) defienden que son las variables de tipo motivacional y personal, como las habilidades o estrategias de afrontamiento y las variables emocionales, las que influyen de una manera más significativa sobre el síndrome de burnout.

\subsection{Concepto de mindfulness}

Para atender a los problemas actuales del alumno universitario, es necesario que el sistema educativo universitario fomente el desarrollo de herramientas que incluyan competencias socioemocionales además del desarrollo de habilidades académicas. Desde el ámbito de la investigación educativa, se ha propuesto el estilo de acción-emoción, 
como una variable motivacional y afectiva de logro, moduladora del rendimiento académico. Existe por tanto un interés creciente en el uso de prácticas de atención plena para promover el crecimiento personal y aumentar el bienestar de los estudiantes universitarios cultivando la conciencia y la comprensión (Dvorakova et al., 2017).

La práctica de la atención plena puede definirse como el estado de estar atento y aceptar lo que está ocurriendo en el presente (Brown y Ryan, 2003). Generalmente al término de atención plena se le conoce como mindfulness. Bishop y colaboradores (2004) consideran que la psicología actual ha adoptado al mindfulness como una técnica para incrementar la consciencia.

La atención plena o mindfulness puede conceptualizarse como la práctica de aceptar y no criticar la actitud ante los pensamientos y sentimientos del momento presente. $\mathrm{Al}$ cultivar la atención plena, los estudiantes pueden aprender técnicas efectivas del manejo del estrés y habilidades de regulación emocional, estando mejor formados para tomar decisiones más saludables (Dvorakova et al., 2017).

Según Kabat-Zinn (2003), mindfulness es la conciencia que surge a través de prestar atención deliberadamente a presentar experiencias sin juicio o preferencia. Es una orientación a la experiencia en la que uno se compromete con una actitud de amabilidad y curiosidad (Nixon et al., 2016). La atención plena, la habilidad de aprender a prestar atención sin juicio, a la experiencia del momento presente, ofrece una manera de mejorar el bienestar en diversas poblaciones. La atención plena según Roberts y Danoff-Burg (2011) es una forma de prestar atención, una conciencia de momento a momento, sin juicio, que implica centrarse deliberadamente en las experiencias del momento presente. Según Kabat-Zinn, en los Estados Unidos, la reducción del estrés basada en la atención plena (MSBR) y las variantes de MBSR son los métodos más utilizados para enseñar meditación de atención plena a los adultos (Greeson et al., 2014). La tradición espiritual oriental sugiere que la atención plena puede ser cultivada a través de la práctica regular de la meditación.

La atención plena o mindfulness comprende dos componentes: la conciencia y la aceptación (Cardaciotto et al., 2008). La conciencia implica una atención a lo que está sucediendo en el momento presente, mientras que la aceptación indica una actitud abierta y sin prejuicios a las experiencias y estímulos actuales. Descubrieron que la aceptación consciente se asociaba negativamente con el estrés percibido, puesto que si hay aceptación es más probable que se dé el bienestar. En la misma línea, Moses y colaboradores (2016) demostraron que la aceptación consciente era la práctica más estrechamente vinculada con el bienestar del estudiante universitario.

\subsection{Influencia del mindfulness en estudiantes universitarios}

Las intervenciones basadas en la atención plena están floreciendo dentro del campo de la salud mental (Baer, 2006; Singh, 2010) encontrándose dos enfoques para ello, la reducción del estrés basada en la atención plena (MBSR) (Kabat-Zinn, 2003) y la terapia cognitiva basada en la atención plena (MBA). Por tanto, los beneficios tanto psicológicos como físicos que propician la atención plena han sido investigados en diferentes estudios.

Kuyken y otros (2013) evaluaron la aceptabilidad y eficacia de un programa de mindfulness para mejorar la salud mental y los factores de bienestar tales como el afrontar tareas sin miedo y con disfrute, la autoestima y la empatía. En la misma línea Franco, De la fuente y Salvador (2011) afirman que el mindfulness es válido para 
promover el crecimiento y auto-realización personal de quiénes lo realizan. Así como Nelis y colaboradores (2009) encontraron que los adultos jóvenes que participaron en un curso intensivo de formación emocional mejoraron sus niveles de inteligencia emocional.

Recientemente, se está discutiendo cada vez más sobre la utilidad del mindfulness en la educación, pero pocas investigaciones han explorado si la atención plena puede influir en las percepciones del entorno educativo. En los últimos tiempos, los enfoques basados en la atención plena también se han aplicado a otros contextos no médicos, como la educación (Hyland, 2009). En esta área se ha explorado la capacidad de observar la experiencia sin juzgar como medio para no sólo mejorar el bienestar y la comunicación, sino también el rendimiento. De la misma manera la atención plena mejora la retención de conocimientos de los estudiantes (Ramsburg y Youmans, 2014), mejora las calificaciones de los estudiantes en Lectura y Ciencias, así como su desempeño en exámenes de algo riesgo (Bakosh et al., 2016).

Investigaciones recientes como la de Dvorakova y colaboradores (2017) demuestran la efectividad de un programa de mindfulness para promover la salud y el bienestar en estudiantes universitarios, aumentando la satisfacción de la vida del estudiante y disminuyendo de manera significativa la depresión y la ansiedad. Forquer y colaboradores (2008) informaron que más de un tercio de los estudiantes necesita más de 30 minutos para dormirse, se despiertan más de una vez por la noche y está cansado durante todo el día. Investigaciones han demostrado que la atención plena puede reducir los trastornos de sueño o el tabaquismo que están relacionados con el estrés (Carlson y Garland, 2005; Davis et al., 2007).

En conjunto, estos estudios de atención plena sugieren que la adquisición y el desarrollo de la práctica de la atención plena se asocia con disminuciones en la angustia emocional, y aumentos de estados más positivos. Actualmente hay programas universitarios en el Reino Unido donde se ofrece formación en mindfulness dentro del currículum educativo (Nixon et. al., 2016), como en universidades de Australia (Lindsay et al., 2015), implementando y evaluando estrategias para apoyar el bienestar de los estudiantes.

Ante esta situación, se ve la importancia que cobra el desarrollo de programas afectivomotivacionales para mejorar la auto-aceptación y realización personal en universitarios. Salami (2010) señala la importancia de diseñar estrategias de promoción y bienestar psicológico en el alumnado como medio, a su vez, de mejorar la calidad de enseñanza. Los resultados prometedores que se alcanzan con la práctica del mindfulness sugieren a las instituciones educativas que lo integren para conseguir resultados beneficiosos en el bienestar emocional, la capacidad y potencial de aprendizaje y en la salud física y mental de quienes lo practican.

La calidad de vida y la salud mental de los estudiantes universitarios es un tema emergente a tener en cuenta cada vez más en un contexto internacional ya que influye directamente en la calidad del proceso de formación de los futuros profesionales y ciudadanos. De igual manera un creciente cuerpo de estudios sobre los beneficios del mindfulness ha comenzado a mostrar su potencial como estrategia de intervención para mejorar la salud mental y el rendimiento en general. Por tanto, contar con resultados de una revisión sistemática sobre la relación específica entre mindfulness, salud y bienestar psicológico del alumno universitario, así como su rendimiento académico, creemos puede ser el comienzo de un cuerpo de estudio interesante como punto de partida de futuras investigaciones. 
Debido a la importancia que está alcanzando el mindfulness en las Ciencias Sociales, la finalidad de este trabajo es llevar a cabo una revisión sistemática de los estudios encontrados en la Web of Science durante los últimos diez años que relaciona el mindfulness con la población universitaria, con el objetivo de constatar el número de investigaciones existentes según el país de procedencia y el año de publicación, extrayendo las características principales de cada uno de los trabajos como son la universidad de procedencia, la muestra seleccionada, el tipo de investigación y los instrumentos de recogida de datos; para finalmente agruparlas en base a los aspectos de mejora del estudiante universitario.

\section{Método}

La revisión bibliográfica de los artículos ha sido llevada a cabo durante los primeros cinco meses de 2017, incluyéndose los trabajos publicados hasta mayo de 2017 prestando mayor atención a aquellos que relacionan el mindfulness con la educación universitaria durante la última década, 2007-2017, con la intención de cuantificar las investigaciones que se han realizado en los últimos años, clasificando éstas según el objetivo de mejora buscado, constatando la necesidad de aumentar las investigaciones en este emergente cuerpo de estudio.

Para la realización de este trabajo, se han tenido en cuenta los artículos publicados en cualquier lengua. Como principal motor de búsqueda se ha empleado la Web of Science (WOS), obteniendo un total de 652 relacionados con la temática de estudio. Se usaron los términos mindfulness, school y education como palabras clave, y como operadores boleanos and $\mathrm{y}$ or.

Se refinó la búsqueda considerando solamente los artículos publicados en los dominios de investigación Social Science al ser el área de conocimiento de los investigadores implicados en el trabajo, dentro del campo de la educación y se tuvo como prioridad las áreas de investigación Education Educational Research y Social Science refinándose a un total de 96 artículos. Para seleccionar la muestra de estudio se establecieron los siguientes criterios:

- Artículos científicos que relacionen el mindfulness con estudiantes universitarios;

- Estudios que consideren el mindfulness como herramienta significativa en la mejora de las capacidades del estudiante universitario.

La aplicación de estos criterios de inclusión se realizó mediante una primera lectura del título y resumen de los 96 trabajos para cumplir el primer criterio. Posteriormente se realizó una lectura pormenorizada del texto completo de los trabajos seleccionados para aplicar el resto de criterios. De tal manera que, al aplicar los criterios conceptuales y metodológicos, se eliminaron 73 artículos.

A partir de lo expuesto en el apartado de procedimiento, se analizaron 96 artículos científicos encontrados en la base de datos wos. Tras considerar y aplicar los criterios de inclusión, la muestra de esta revisión sistemática se corresponde a 23 artículos científicos. 


\section{Resultados}

A continuación, presentamos los resultados encontrados en nuestra revisión sistemática, se analiza la evolución de la producción científica y las principales características de los estudios analizados.

\subsection{Evolución de la producción científica}

Durante el periodo 2007-2017 se han publicado un total de 96 artículos relacionados con el mindfulness y la educación, incluidos en las categorías de Education Educational y Social Science en WOS. Sin embargo, los estudios que vinculan el mindfulness con el estudiante universitario descienden a un total de 23 artículos correspondiéndose con el 23,9\% de la totalidad de artículos producidos sobre esa temática. En la figura 1 se compara la producción total de estudios por año de wos, con aquellos que forman el cuerpo base de la investigación. La producción de estudios que versan sobre la relación existente entre el mindfulness y los estudiantes universitarios es creciente sobre todo a partir del 2014, aunque a su vez hemos de destacar un leve declive en la producción desde el 2016 tal y como podemos observar en la figura 1.

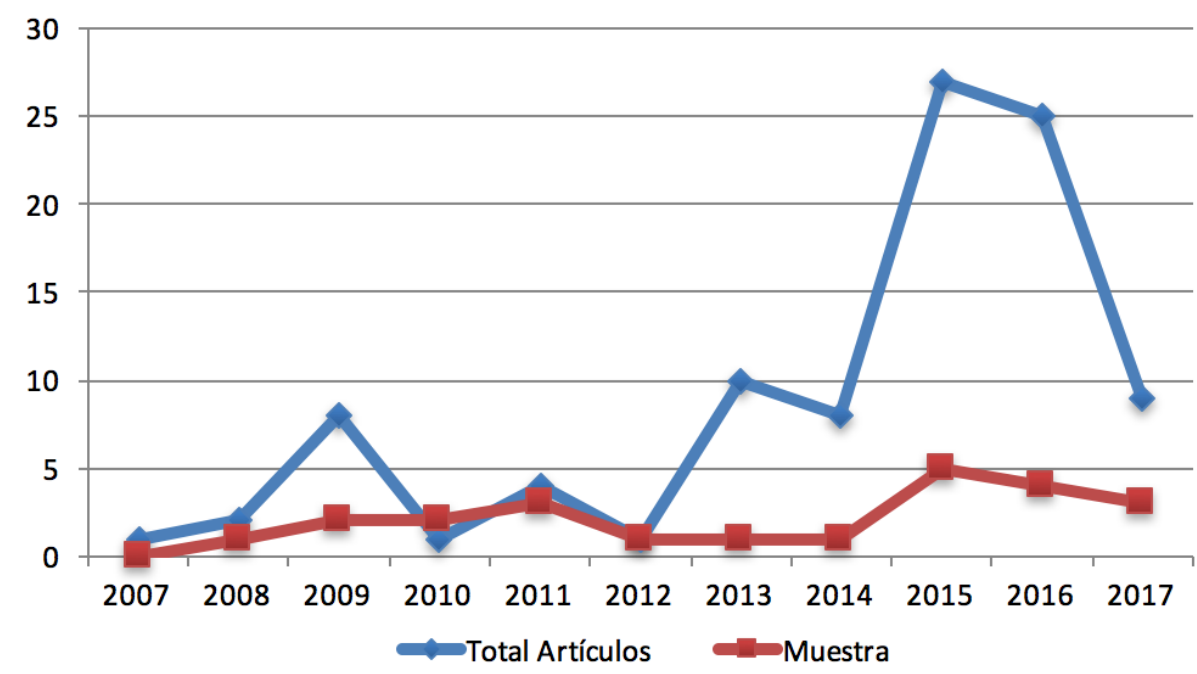

Figura 1. Comparación de la producción de artículos científicos Fuente: Elaboración propia.

La producción de artículos que abordan el mindfulness como un factor influyente en el desarrollo del estudiante universitario en la última década es ascendente, como se puede apreciar en la figura 1, siendo este incremento más notable en el año $2015(\mathrm{~N}=5)$. Sin embargo, son muchos menos los trabajos dedicados al estudiante universitario que, al resto de las etapas educativas, lo que se vincula con el objetivo de nuestra investigación.

\subsection{Características comunes de los estudios sobre mindfulness y estudiantes universitarios}

Como ya hemos indicado, la muestra de esta revisión sistemática se compone de 23 artículos en los que han participado un total de 3.835 alumnos universitarios, tal y como se puede observar en el cuadro 1. 
Cuadro 1. Síntesis de estudios que constituyen el cuerpo base de la revisión sistemática

\begin{tabular}{|c|c|c|c|c|c|}
\hline & AUTOR/ES & País & $\mathbf{N}$ & $\begin{array}{c}\text { TIPO DE } \\
\text { INVESTIGACIÓN }\end{array}$ & INSTRUMENTOS \\
\hline 1 & $\begin{array}{l}\text { Dvorakova, } \\
\text { Kishida, Li, } \\
\text { Elavsky, Broderick, } \\
\text { Agrusti, y } \\
\text { Greenberg (2017) }\end{array}$ & $\begin{array}{l}\text { Estados } \\
\text { Unidos }\end{array}$ & 109 & Transversal & $\begin{array}{l}\text { “PHQ” “GAD” “SWL” } \\
\text { “MAAS” “SCS” “SCCR” } \\
\text { “CS” “PSPI” "YAAPST" }\end{array}$ \\
\hline 2 & $\begin{array}{l}\text { Sheikhzadeh y } \\
\text { Khatami (2017a) }\end{array}$ & Irán & 220 & Transversal & $\begin{array}{l}\text { “CCTT” “MAAS” } \\
\text { “TOEFL TEST” }\end{array}$ \\
\hline 3 & $\begin{array}{l}\text { Sheikhzadeh y } \\
\text { Khatami (2017b) }\end{array}$ & Irán & 220 & Transversal & $\begin{array}{c}\text { “OPT” "MAAS” “TOEFL } \\
\text { TEST" } \\
\end{array}$ \\
\hline 4 & $\begin{array}{l}\text { Bore, Pittolo, } \\
\text { Kirby, Dluzewska y } \\
\text { Marlin (2016) }\end{array}$ & Australia & 150 & Longitudinal & $\begin{array}{c}\text { “K 10” “GHQ-12” “BSI” } \\
\text { “GSI” "WEMWBS” } \\
\text { "BRS” }\end{array}$ \\
\hline 5 & $\begin{array}{l}\text { Moses, Bradley, y } \\
\text { O'Callaghan (2016) }\end{array}$ & Australia & 203 & Transversal & $\begin{array}{c}\text { "Escala de } \\
\text { mindfulness } \\
\text { Philadelphia" }\end{array}$ \\
\hline 6 & $\begin{array}{l}\text { Nixon, McMurtry, } \\
\text { Craig, Nevejan y } \\
\text { Regan-Addis (2016) }\end{array}$ & Escocia & 63 & Longitudinal & “ED505 mindfulness) \\
\hline 7 & $\begin{array}{l}\text { Xu, Wu, Zhao, } \\
\text { Chen, Xia, Li, y } \\
\text { Zhong (2016) }\end{array}$ & China & $431 / 231$ & Longitudinal & "DREEM-C" “KIMS-C” \\
\hline 8 & $\begin{array}{l}\text { Gulgez y Gunduz } \\
(2015)\end{array}$ & Turquía & 18 & Transversal & “DERS" \\
\hline 9 & $\begin{array}{l}\text { Lindsay, Kirby, } \\
\text { Dluzewska, y } \\
\text { Campbell (2015) }\end{array}$ & Australia & 75 & Transversal & “MSLQ” \\
\hline 10 & $\begin{array}{l}\text { Pegalajar y López } \\
\text { Hernáez (2015) }\end{array}$ & España & 183 & Transversal & “AURE” \\
\hline 11 & $\begin{array}{l}\text { Slonim, Kienhuis Di } \\
\text { Benedetto y Reece } \\
(2015)\end{array}$ & Australia & 207 & Transversal & $\begin{array}{c}\text { “HPLPII” “FFMQ” } \\
\text { “DASS” }\end{array}$ \\
\hline 12 & $\begin{array}{l}\text { Van Dijk, Lucassen } \\
\text { y Speckens (2015) }\end{array}$ & Holanda & $95 / 167$ & Longitudinal & $\begin{array}{l}\text { “BSI” “NEO-FFI” } \\
\text { "FFMQ" “IBI" }\end{array}$ \\
\hline 13 & $\begin{array}{l}\text { Greeson, Juberg, } \\
\text { Maytan, James, y } \\
\text { Rogers (2014) }\end{array}$ & $\begin{array}{l}\text { Estados } \\
\text { Unidos }\end{array}$ & 90 & Transversal & $\begin{array}{c}\text { “PSS” “MOS SLPQ” } \\
\text { “CAMS-R” “SCS” “GQ- } \\
6 \text { " }\end{array}$ \\
\hline 14 & $\begin{array}{l}\text { De Vibe, Solhaug, } \\
\text { Tyssen, Friborg, } \\
\text { Rosenvinge, Sorlie } \\
\text { y Bjorndal (2013) }\end{array}$ & Noruega & 288 & Transversal & $\begin{array}{c}\text { “GHQ12" “MBI” } \\
\text { "PMSS” “SWB” "FFMQ” }\end{array}$ \\
\hline 15 & Akin (2012) & Turquía & 299 & Transversal & $\begin{array}{c}\text { Escala de } \\
\text { autocompasión “ATQ" }\end{array}$ \\
\hline 16 & $\begin{array}{l}\text { Grinnell, Greene, } \\
\text { Melanson, } \\
\text { Blissmer, y Lofgren } \\
(2011)\end{array}$ & $\begin{array}{l}\text { Estados } \\
\text { Unidos }\end{array}$ & 75 & Transversal & $\begin{array}{l}\text { “MAAS” “WRBQ” “FV” } \\
\text { "IPAQ-S” }\end{array}$ \\
\hline 17 & $\begin{array}{l}\text { Hewett, Ransdell, } \\
\text { Gao, Petlichkoff, y } \\
\text { Lucas (2011) }\end{array}$ & $\begin{array}{l}\text { Estados } \\
\text { Unidos }\end{array}$ & 51 & Transversal & “PAR-Q" “FFMQ” “PSS” \\
\hline 18 & $\begin{array}{l}\text { Roberts y Danoff- } \\
\text { Burg (2011) }\end{array}$ & $\begin{array}{l}\text { Estados } \\
\text { Unidos }\end{array}$ & 553 & Transversal & $\begin{array}{c}\text { “FFMQ” “PSQI” “WLI” } \\
\text { "BES” }\end{array}$ \\
\hline
\end{tabular}


Cuadro 1bis. Síntesis de estudios que constituyen el cuerpo base de la revisión sistemática (Continuación)

\begin{tabular}{|c|c|c|c|c|c|}
\hline & AUTOR/ES & PAís & $\mathbf{N}$ & $\begin{array}{c}\text { TIPO DE } \\
\text { INVESTIGACIÓN }\end{array}$ & INSTRUMENTOS \\
\hline 19 & Akin (2010) & Turquía & 338 & Transversal & $\begin{array}{c}\text { Escala de } \\
\text { autocompasión, Escala } \\
\text { de distorsiones } \\
\text { cognitivas } \\
\text { interpersonales }\end{array}$ \\
\hline 20 & $\begin{array}{l}\text { Arias, Justo y } \\
\text { Manas (2010) } \\
\end{array}$ & España & 38 & Transversal & “ECE” “MBI-GS” \\
\hline 21 & Akin (2009) & Turquía & 755 & Transversal & $\begin{array}{c}\text { Escala de } \\
\text { autocompasión "SAS" }\end{array}$ \\
\hline 22 & $\begin{array}{l}\text { Hassed, De Lisle, } \\
\text { Sullivan y Pier } \\
(2009)\end{array}$ & Australia & 148 & Transversal & $\begin{array}{c}\text { "SCL-9OR)" “DEP” } \\
\text { “ANX” "HOS" "WHOQOL } \\
\text { BREF2" }\end{array}$ \\
\hline 23 & Akin (2008) & Turquía & 646 & Transversal & $\begin{array}{l}\text { “AGOS” Escala de } \\
\text { autocompasión }\end{array}$ \\
\hline
\end{tabular}

Fuente: Elaboración propia.

Para extraer los datos, se han tenido en cuenta el siguiente proceso de codificación: (1) autor/res; (2) año de publicación; (3) Lugar de procedencia de la universidad; (4) muestra; (5) tipo de investigación y (6) instrumentos de recogida de la información.

Tras la revisión podemos constatar cómo las investigaciones realizadas sobre el beneficio del mindfulness en estudiantes universitarios son estudios de corte transversal y longitudinal que utilizan diversos instrumentos de recogida de datos según el tipo de investigación. Los artículos que constituyen el cuerpo base de nuestro trabajo, han desarrollado estudios que relacionan el mindfulness con la etapa universitaria. Por ello, el cuadro 2 muestra aquellos países donde se han realizado las investigaciones. Entre nueve países se encuentran las 23 investigaciones que conforman la muestra de este estudio de revisión sistemática. Los países donde mayor es el número de estudios que relacionan mindfulness y universidad es en Estados Unidos $(\mathrm{N}=5)$, Australia $(\mathrm{N}=5)$ y Turquía $(\mathrm{N}=5)$, a los cuales se les asocia el 21,7\% de estudios albergados. También en países como Escocia, España, China, Holanda y Noruega se ha desarrollado al menos una investigación sobre mindfulness y universidad.

Cuadro 2. Países donde se desarrolla las investigaciones del cuerpo base de investigación

\begin{tabular}{lcc}
\hline \multicolumn{1}{c}{ PAÍ́ } & No $^{\circ}$ DE ESTUDIOS & PORCENTAJE \\
\hline Estados Unidos & 5 & $21,7 \%$ \\
Irán & 2 & $8,6 \%$ \\
Australia & 5 & $21,7 \%$ \\
Escocia & 1 & $4,3 \%$ \\
China & 1 & $4,3 \%$ \\
Turquía & 5 & $21,7 \%$ \\
España & 2 & $8,6 \%$ \\
Holanda & 1 & $4,3 \%$ \\
Noruega & 1 & $4,3 \%$ \\
\hline Total & 23 & $100,0 \%$ \\
\hline
\end{tabular}

Fuente: Elaboración propia. 
Resulta pertinente dividir los estudios seleccionados considerando el objeto de estudio hacia el que van dirigidos, tal y como se muestran en el cuadro 3. Una mayoría de los estudios se han centrado en relacionar el mindfulness con la mejora de la salud y el bienestar psicológico del estudiante universitario encontrándose un total de 18 investigaciones. Seguidamente nos encontramos un total de 4 estudios que se centran en relacionar el mindfulness con la mejora del rendimiento y los resultados académicos. Por último, hay dos investigaciones donde proponen la inclusión del mindfulness dentro del currículum universitario.

Cabe destacar que es en el primer grupo, las investigaciones que relacionan el mindfulness con la mejora de la salud y el bienestar psicológico donde se encuentran los artículos más citados dentro de la wOS, con un total de 183 citas.

Cuadro 3. Hacia qué aspectos de mejora van dirigidos los estudios del cuerpo base de investigación

\begin{tabular}{|c|c|c|c|c|}
\hline MEJORA & $\mathbf{N}^{\circ}$ & PAÍS & AÑo & No CITAS EN WOS \\
\hline \multirow{18}{*}{$\begin{array}{l}\text { Mejora de la salud y el bienestar } \\
\text { psicológico del estudiante universitario }\end{array}$} & 1 & Estados Unidos & 2017 & $\mathrm{O}$ \\
\hline & 4 & Australia & 2016 & $\mathrm{O}$ \\
\hline & 5 & Australia & 2016 & $\mathrm{O}$ \\
\hline & 8 & Turquía & 2015 & $\mathrm{O}$ \\
\hline & 10 & España & 2015 & $\mathrm{O}$ \\
\hline & 11 & Australia & 2015 & 5 \\
\hline & 12 & Holanda & 2015 & 5 \\
\hline & 13 & Estados Unidos & 2014 & 12 \\
\hline & 14 & Noruega & 2013 & 32 \\
\hline & 15 & Turquía & 2012 & 10 \\
\hline & 16 & Estados Unidos & 2012 & $\mathcal{Q}$ \\
\hline & 17 & Estados Unidos & 2011 & 7 \\
\hline & 18 & Estados Unidos & 2011 & 12 \\
\hline & 19 & Turquía & 2010 & 10 \\
\hline & 20 & España & 2010 & 1 \\
\hline & 21 & Turquía & 2009 & 12 \\
\hline & 22 & Australia & 2009 & 67 \\
\hline & 23 & Turquía & 2008 & 13 \\
\hline \multirow{4}{*}{$\begin{array}{l}\text { Mejora en el rendimiento académico y } \\
\text { en los resultados académicos }\end{array}$} & 2 & Irán & 2017 & $\mathrm{O}$ \\
\hline & 3 & Irán & 2017 & $\mathrm{O}$ \\
\hline & 7 & China & 2016 & 1 \\
\hline & 20 & España & 2010 & 1 \\
\hline \multirow{2}{*}{$\begin{array}{l}\text { Propuesta de inclusión del mindfulness } \\
\text { en los currículums universitarios }\end{array}$} & 6 & Escocia & 2016 & $\mathrm{O}$ \\
\hline & 9 & Australia & 2015 & $\mathrm{O}$ \\
\hline
\end{tabular}

Fuente: Elaboración propia.

\section{Discusión}

La revisión sistemática que se ha desarrollado pone de manifiesto que el número de artículos que estudia el tratamiento de la atención plena o mindfulness como factor esencial para un desarrollo integral del alumnado universitario, es cada vez mayor. Se han registrado un total de 23 investigaciones mostrando cómo van en aumento en la actualidad, siendo países de habla inglesa principalmente (Estados Unidos y Australia), 
los responsables del mayor número de estudios junto con Turquía, donde el principal aspecto de mejora está enfocado a la salud y el bienestar psicológico del estudiante.

En cuanto a los estudios clasificados en base a los aspectos de mejora a los que van dirigidos, creemos conveniente discutir los resultados según las tres categorías encontradas.

\section{Mejora de la salud y el bienestar psicológico del estudiante universitario}

Nos encontramos que el grueso de las investigaciones, están enfocadas en esta categoría, con un total de 18 artículos, siendo países de habla inglesa principalmente los responsables de la mayoría de las investigaciones realizadas. En estos trabajos se prioriza el bienestar psicológico y la salud mental del estudiante, frente al rendimiento académico.

En la investigación realizada por Dvorakova y colaboradores (2017), en 109 estudiantes universitarios de primer año se evaluó la efectividad de un programa de mindfulness para promover la salud y el bienestar de los alumnos. Los resultados obtenidos muestran un aumento significativo en la satisfacción de la vida de los estudiantes y una disminución en la depresión y ansiedad. Encontramos datos similares en otros estudios como el de Bore y colaboradores (2016), donde se reafirma una mejora en el malestar psicológico y aumento del bienestar del estudiante universitario. Además, el estudio de Moses, Bradley y O'Callaghan (2016), en una muestra de 206 universitarios los hallazgos sugieren que la práctica de la atención plena, del mindfulness puede tener beneficios de salud mental con mayor significatividad que los beneficios obtenidos a través de actividades más tradicionales como la dieta y el ejercicio físico. Así como la investigación de Guldez y Gunduz (2015) que confirman la efectividad de un programa de atención plena para reducir las dificultades de los estudiantes universitarios en la regulación emocional. Respecto al tipo de estudios universitarios, Pegalajar y López Hernáez (2015) se centraron en 183 estudiantes del grado de educación infantil donde se aplica un programa de mindfulness, muestran unos niveles muy favorables de autorrealización y desarrollo personal. De igual manera, Slonim y colaboradores (2015) realizaron similares investigaciones centradas en 207 estudiantes de medicina en Australia, señalando el potencial del autocuidado y la atención plena para disminuir la aflicción y la angustia psicológica, de la misma manera que la investigación realizada por Van Dijk, Lucassen y Speckens (2015) en estudiantes de medicina holandeses.

Las investigaciones de Greeson y otros (2014) demuestran que un programa de atención mental en 90 alumnos universitarios es eficaz para reducir los síntomas de estrés, mejorar el bienestar psicológico y promover el sueño. De la misma manera los estudios de De Vibe y colaboradores (2013) sobre una muestra de 288 alumnos universitarios constatan que un programa de entrenamiento de atención plena redujo la angustia mental y mejoró el bienestar de los estudiantes de medicina y psicología de dos universidades noruegas.

En los estudios de Grinnell y otros (2011), se observaron correlaciones entre la atención plena y los comportamientos relacionados con el peso, comprobándose que la atención plena puede afectar el estado de salud de los estudiantes universitarios de primer año, particularmente respecto a las medidas de comportamiento que afectan el estado del peso. 
En la misma línea los resultados de la investigación realizada por Hewett y colaboradores (2011) demuestran que un programa de yoga Bikram afectó positivamente la salud psicológica y física en 51 estudiantes universitarios de Estados Unidos. De igual manera Roberts y Danoff-Gurg (2011) en su estudio con 553 estudiantes universitarios demostraron un vínculo entre la atención y las percepciones y comportamientos de salud en una población universitaria. Los resultados sugieren que la atención plena o mindfulness está relacionada con la disminución del estrés, contribuyendo a aumentar las percepciones positivas de salud y comportamientos de salud.

Investigaciones similares como la de Arias, Justo y Mañas (2010), confirman que un programa de intervención de mindfulness produjo mejoras significativas en el cansancio y agotamiento emocional en alumnos universitarios de Psicología. Hassed y otros (2009) en sus investigaciones con 148 estudiantes universitarios de medicina constataron la influencia positiva de un programa de atención plena sobre el bienestar de dichos estudiantes.

El número de investigaciones que relaciona el mindfulness con la salud mental y el bienestar psicológico va en aumento como se desprende de los resultados anteriores. Los estudios en lengua inglesa son muy abundantes en relación al resto de las lenguas.

\section{Mejora en el rendimiento y en los resultados académicos}

En cuanto a los efectos del mindfulness o atención plena sobre el rendimiento académico, encontramos cuatro estudios realizados en Irán, China y España en los últimos cinco años, donde se constata la influencia positiva del mindfulness sobre los resultados académicos. Así, resalta la investigación de Sheikhzadeh y Khatami (2017a, 2017b) en estudiantes universitarios iraníes donde se demostró la correlación positiva entre la atención plena y la comprensión lectora y rendimiento académico. La investigación de Xu y colaboradores (2016) relacionan la atención plena con una mayor satisfacción en la percepción del entorno educativo, del ambiente de aprendizaje en estudiantes de medicina en China, mejorando el clima escolar y, por tanto, influyendo positivamente en los resultados académicos. En la misma línea los estudios de Arias, Justo y Mañas (2010), corroboran que un programa de mindfulness produce cambios en el cansancio emocional y por tanto influye positivamente en la capacidad de concentración del estudiante en su actividad académica.

Cabe destacar el número tan reducido de investigaciones encontradas durante la última década dedicadas a la mejora del rendimiento académico a través de la aplicación del mindfulness, frente a aquellas dedicadas a la mejora de la salud mental.

\section{Propuesta de inclusión del mindfulness en el currículum universitario}

A tenor de la importancia del mindfulness como estrategia para la regulación emocional de los estudiantes universitarios, así como para la mejora del rendimiento académico, encontramos investigaciones como la de Nixon y otros (2016), que proponen la inclusión del mindfulness en el currículum universitario. Así, en diversos programas de postgrado del Reino Unido, se ofrece formación en mindfulness desde una perspectiva de ciencia social en lugar de la perspectiva clínica. Actualmente hay estudios de grado con una duración de cuatro años. En esta línea Moses, Bradley y O’Callaghan (2016) proponen que se le ofrezcan al estudiante universitario cursos académicos que integren el entrenamiento de la atención plena en el currículo principal. Lindsay y otros (2015) tras los resultados en sus estudios, proponen actualizar en la facultad de derecho de Australia 
los estudios para incluir un currículo de autogestión. Greeson y colaboradores (2014) apoyan esta idea al considerar la atención plena una intervención viable para los centros de consejería estudiantil que busquen proporcionar intervenciones rentables para los estudiantes que sufren de niveles inmanejables de estrés. A este respecto, sólo dos investigaciones cuyo objetivo es la inclusión del mindfulness en el currículum universitario fueron publicadas en el año 2015 y 2016.

\section{Conclusiones}

Por consiguiente, sin atendemos a la fundamentación teórica revisada y a las conclusiones arrojadas por las investigaciones recogidas que componen el cuerpo base de este trabajo, podemos decir que la mayoría de investigaciones de la última década utilizan el mindfulness como herramienta fundamental para la mejora del estado de bienestar y salud mental indispensable en el estudiante universitario, quedando en un segundo plano por el bajo número de investigaciones, aquellos trabajos donde el mindfulness mejora el rendimiento académico y se incluye dentro del currículum universitario.

En relación a los estudios que se han llevado en diferentes países, encontramos un total de 9, destacando la labor de Turquía, Australia y Estados Unidos con un total de 5 investigaciones en cada caso. El resto de los países tienes un promedio de 1-2 estudios. En este sentido, sería interesante diseñar más intervenciones que estudien la influencia del mindfulness en el ámbito universitario.

A partir de los resultados obtenidos, se propone llevar a cabo programas de atención plena o mindfulness dentro del contexto universitario para la mejora del bienestar psicológico y del rendimiento académico como estrategia de intervención a nivel institucional, mejorando y ampliando los recursos de ayuda y orientación al estudiante, en el ámbito de la mejora de las competencias de regulación personal. El actual panorama universitario requiere que la implicación de los diferentes agentes educativos vaya más allá de la mera transmisión de contenidos académicos y aborde nuevos enfoques para la mejora y el bienestar integral de los universitarios.

Una de las limitaciones de este estudio ha sido el periodo de tiempo seleccionado, ya que se podría abarcar una década más para poder conocer su evolución con mayor profundidad. Aun así, el proceso de revisión exhaustivo al que han sido sometidos los artículos seleccionados garantiza la calidad de los estudios seleccionados.

Son necesarias futuras investigaciones donde se centren en la aplicación del mindfulness para conocer las variables que son susceptibles de mejora y cambio y cuáles no, para comparar diferentes programas de mindfulness entre sí sobre diferentes variables. Se hace evidente la necesidad de utilizar este tipo de programas con diferentes perfiles universitarios y comprobar el logro académico obtenido.

\section{Referencias}

Akin, A. (2008). Self-compassion and achievement goals: A structural equation modeling approach. Eurasian Journal of Educational Research, 8(31), 1-15.

Akin, A. (2009). Self-compassion and submissive behavior. Education and Science, 34(152), 138147 . 
Akin, A. (2010). Self-compassion and interpersonal cognitive distortions. Hacettepe University Journal of Education, 39, 1-9.

Akin, A. (2012). Self-compassion and automatic thoughts. Hacettepe University Journal of Education, 42, 1-10.

Arias, J. D., Justo, C. F. y Mañas, I. M. (2010). Results of a program on mindfulness on the emotional situation of university students. Estudios Sobre Educacion, 19, 31-52.

Bakosh, L. S., Snow, R. M., Tobias, J. M., Houlihan, J. L. y Barbosa-Leiker, C. (2016). Maximizing mindful learning: Mindful awareness intervention improves elementary school students' quarterly grades. Mindfulness, 7, 59-67. https://doi.org/10.1007/s12671015-0387-6

Baer, R. (Ed.). (2006). Mindfulness based treatment approaches: Clinician's guide to evidence based and applications. Londres: Elsevier.

Bishop, S. R., Lau, M., Shapiro, S., Carlson, L., Anderson, N. y Cardomy, J. (2004). Mindfulness: A proposed operational definition. Clinical Psychology: Science and Practice, 10, 230-241. https://doi.org/10.1093/clipsy.bpho77

Bore, M., Pittolo, C., Kirby, D., Dluzewska, T. y Marlin, S. (2016). Predictors of psychological distress and well-being in a sample of Australian undergraduate students. Higher Education Research E $\quad$ Development, 35(5), 869-880. https://doi.org/10.1080/07294360.2016.1138452

Brown, K. W. y Ryan, R. M. (2003). The benefit being present: Mindfulness an its role in psychological well-being. Journal of Personality and Social Psychology, 84(4), 822-848. https://doi.org/10.1037/0022-3514.84.4.822

Caballero, C., Abello, R. y Palacio, J. (2007). Relación del burnout y el rendimiento académico con la satisfacción frente a los estudios en estudiantes universitarios. Avances en Psicología Latinoamericana, 25(2), 98-111.

Cardaciotto, L., Herbert, J. D., Forman, E. M., Moitra, E. y Farrow, V. (2008). The assessment of present-moment awareness and acceptance: The Philadelphia mindfulness scale. Assessment, 15(2), 204-223. https://doi.org/10.1177/1073191107311467

Carlson, L. E. y Garland, S. N. (2005). Impact of mindfulness based stress reduction (MBSR) on sleep, mood, stress, and fatigue symptoms in cancer outpatients. International Journal of Behavioral Medicine, 12, 278-285. https://doi.org/10.1207/s 15327558ijbm1204_9

Davis, J. M., Fleming, M. F., Bonus, K. A. y Baker, T. B. (2007) A pilot study on mindfulness based stress reduction for smokers. BMC Complementary and Alternative Medicine, 7, 1-7. https://doi.org/10.1186/1472-6882-7-2

De Vibe, M., Solhaug, I., Tyssen, R., Friborg, O., Rosenvinge, J. H. y Sorlie, T. (2013). Mindfulness training for stress management: a randomised controlled study of medical and psychology students. BMC Medical Education, 13, 1-14. https://doi.org/10.1186/1472-6920-13-107

Dlaska, A. (2013). The role of foreign language programmes in internationalising learning and teaching in higher education. Teaching in Higher Education, 18(3), 260-271. https://doi.org/10.1080/13562517.2012.696538

Dvorakova, K., Kishida, M., Li, J., Elavsky, S., Broderick, P. C., Agrusti, M. R. y Greenberg, M. T. (2017). Promoting healthy transition to college through mindfulness training with first-year college students: Pilot randomized controlled trial. Journal of American College Health, 65(4), 259-267. https://doi.org/10.1080/07448481.2017.1278605 
Forquer, L. M., Camden, A., Gabriau, K. y Johnson, C. (2008). Sleep patterns of college students at a public university. Journal of American College Health, 56, 563-565. https://doi.org/10.3200/JACH.56.5.563-565

Franco, C., De la Fuente, M. y Salvador, M. (2011). Impacto de un programa de entrenamiento en conciencia plena (mindfulness) en las medidas del crecimiento y la autorealización personal. Psicothema, 23(1), 58-65.

Garcés de los Fayos, E. J. (1995). Burnout en niños y adolescentes: Un nuevo síndrome en psicopatología infantil. Psicothema, 7(1), 33-40.

González, N. (2008). Prevalencia del estrés en la satisfacción laboral de los docentes universitarios. Revista Electrónica de Humanidades, Educación y Comunicación Social, 3(4), 6889 .

González, M. T. y Landero, R. (2007). Escala de cansancio emocional (ECE) para estudiantes universitarios. Propiedades psicométricas en una muestra de México. Anales de Psicología, 23(2), 253-257.

Greeson, J. M., Juberg, M. K., Maytan, M., James, K. y Rogers, H. (2014). A randomized controlled trial of Koru: A mindfulness program for college students and other emerging adults. Journal of American College Health, 62(4), 222-233. https://doi.org/10.1080/07448481.2014.887571

Grinnell, S., Greene, G., Melanson, K., Blissmer, B. y Lofgren, I. E. (2011). Anthropometric and behavioral measures related to mindfulness in college students. Journal of American College Health, 59(6), 539-545. https://doi.org/10.1080/07448481.2011.555932

Gulgez, O. y Gunduz, B. (2015). The effect of dialectical behavior therapy based emotion regulation program on decreasing the emotion regulation difficulties of university students. Cukurova University Faculty of Education Journal, 44(2), 191-208.

Hassed, C., de Lisle, S., Sullivan, G. y Pier, C. (2009). Enhancing the health of medical students: Outcomes of an integrated mindfulness and lifestyle program. Advances in Health Sciences Education, 14(3), 387-398. https://doi.org/10.1007/s10459-008-9125-3

Hewett, Z. L., Ransdell, L. B., Gao, Y., Petlichkoff, L. M. y Lucas, S. (2011). An examination of the effectiveness of an 8-week bikram yoga program on mindfulness, perceived stress, and physical fitness. Journal of Exercise Science \& Fitness, 9(2), 87-92. https://doi.org/10.1016/S1728-869X(12)60003-3

Hyland, T. (2009). Mindfulness and the therapeutic function of education. Journal of Philosophy of Education, 43, 119-131. https://doi.org/10.1111/j.1467-9752.2008.00668.x

Kabat-Zinn, J. (2003). Mindfulness-based interventions in context: Past, present, and future.

Clinical Psychology: Science and Practice, 10, 144-156.

https://doi.org/10.1093/clipsy.bpgo16

Kuyken, W., Weare, K., Ukoumunne, O. C., Lewis, R., Motton, N., Burnett, R., Cullen, C., Hennelly, S. y Huppert, F. (2013). Effectiveness of the mindfulness in schools programme: Non-randomised controlled feasibility study. The British Journal of Psychiatry, 2, 1-6.

Lindsay, K., Kirby, D., Dluzewska, T. y Campbell, S. (2015). Oh, the places you'll go! Newcastle law school's partnership interventions for well-being in first year law. Journal of Learning Design, 8(2), 11-2 1. https://doi.org/10.5204/jld.v8i2.239

Martínez, I. M., Márqués, A., Salanova, M. y Lópes da Silva, A. (2002). Burnout en estudiantes universitarios de España y Portugal. Un estudio transversal. Ansiedad y Estrés, 8(1), 13-23. 
Moses, J., Bradley, G. L. y O'Callaghan, F. V. (2016). When college students look after themselves: Self-care practices and well-being. Journal of Student Affairs Research and Practice, 53(3), 346-359. https://doi.org/10.1080/19496591.2016.1157488

Nelis, N., Quoidbach, J., Mikolajczak, M. y Hansenne, M. (2009). Increasing emotional intelligence: How it is possible? Personality and Individual Differences, 47, 36-41. https://doi.org/10.1016/j.paid.2009.01.046

Nixon, G., McMurtry, D., Craig, L., Nevejan, A. y Regan-Addis, H. (2016). Studies in mindfulness: Widening the field for all involved in pastoral care. Pastoral Care in Education, 34(3), 167-183. https://doi.org/10.1080/02643944.2015.1127990

Paredes, O. L. y Sanabria-Ferrand, P. A. (2008). Prevalencia del síndrome de burnout en residentes de especialidades médico-quirúrgicas, su relación con el bienestar psicológico y con variables sociodemográficas y laborales. Revista de la Facultad de Medicina, 16(1), 2532.

Pegalajar, M. C. y López Hernáez, L. (2015). Emotional competences in the childhood education teacher training. REICE. Revista Iberoamericana sobre Calidad Eficacia y Cambio en Educacion, 13(3), 95-106.

Ramsburg, J. T. y Youmans, R. J. (2014). Meditation in the higher-education classroom: Meditation training improves student knowledge retention during lectures. Mindfulness, 5, 14-31. https://doi.org/10.1007/s12671-013-0199-5

Ryan, R. y Deci, E. (2001). On happiness and human potentials: A review of research on hedonic and edaimonic well-being. Annual Review of Psychology, 52, 141-166. https://doi.org/10.1146/annurev.psych.52.1.141

Roberts, K. C. y Danoff-Burg, S. (2011). Mindfulness and health behaviors: Is paying attention good for you? Journal of American College Health, 59(3), 165-173. https://doi.org/10.1080/07448481.2010.484452

Salami, S. O. (2010). Emotional intelligence, self-efficacy, psychological well-being and student's attitudes: Implications for quality implications. European Journal of Educational Studies, $2(3), 247-257$.

Sheikhzadeh, E. y Khatami, M. (2017a). Critical thinking, mindfulness and academic achievement among Iranian EFL learners. Modern Journal of Language Teaching Methods, 7(3), 281-294.

Sheikhzadeh, E. y Khatami, M. (2017b). The possible relationship between mindfulness and academic achievement among Iranian EFL learners. Modern Journal of Language Teaching Methods, 7(2), 43-48.

Singh, N. N. (2010). Mindfulness: A finger pointing to the moon. Mindfulness,1(1), 1-3. https://doi.org/10.1007/s12671-010-0009-2

Slonim, J., Kienhuis, M., Di Benedetto, M. y Reece, J. (2015). The relationships among self-care, dispositional mindfulness, and psychological distress in medical students. Medical Education Online, 20, 27-42. https://doi.org/10.3402/meo.v20.27924

Stallman, H. (2008). Prevalence of psychological distress in university student. Australian Family Physician, 37(8), 673-677.

Van Dijk, I., Lucassen, P. y Speckens, A. (2015). Mindfulness training for medical students in their clinical clerkships: Two cross-sectional studies exploring interest and participation. BMC Medical Education, 15, 31-56. https://doi.org/10.1186/s12909-015-0302-9

Xu, X., Wu, D. X., Zhao, X. H., Chen, J. X., Xia, J. y Li, M. L. (2016). Relation of perceptions of educational environment with mindfulness among Chinese medical students: a 
longitudinal study. Medical Education Online, 21, 55-78. https://doi.org/10.3402/meo.v2 1.30664

\section{Breve CV de los autores}

\section{Virginia Viciana}

Profesora Titular en el Departamento de Didáctica de la Expresión Corporal de la Facultad de Educación de la Universidad de Granada. Doctora en Educación Física. Licenciada en Ciencias de la Actividad Física y del Deporte. Investigadora del grupo de investigación HUM-238 a través del cual se han desarrollado múltiples tareas de investigación, innovación y transferencia. Autora de ocho libros de reconocido prestigio dentro del campo de la educación física publicados en el grupo editorial "Inde", así como de un total de 21 artículos en revistas científicas, algunos de ellos indexados en la Web of Science y Scopus. Ha participado en más de una treintena de eventos de divulgación científica, como congresos internacionales y jornadas de investigación, mediante ponencias y comunicaciones. ORCID ID: 0000-0003-2711-5572. Email: vviciana@ugr.es

\section{Andrés B. Fernández Revelles}

Profesor Contratado Doctor impartiendo docencia en la Universidad de Granada en las titulaciones de Grado en Ciencias de la Actividad Física y del Deporte, y en el Máster Universitario en Investigación en Actividad Física y Deporte con mención de calidad por la ANECA. Tópico de investigación: Juegos motores / Motor games and active games. Entre sus publicaciones más relevantes se encuentran: "Universidad o mercado laboral: preferencias de los jóvenes a su futuro al terminar los estudios de bachillerato y formación profesional", "Correlación en triatlón masculino entre fases y resultado final en los JJOO de Sídney 2000", "Cultural background shapes spatial reference frame proclivity" y "ABFR-Index: Correlation between Soccer scientific production and ranking”. ORCID ID: 0000-0002-8089-650X. Email: abfr@ugr.es

\section{Marta Linares}

Profesora del Departamento de Enfermería de la Facultad de Ciencias de la Salud de Melilla. Universidad de Granada. Vicedecana de Investigación, Innovación y Posgrado. Máster en Antropología Física y Forense y en Cuidados en Dependencia y Procesos de Fin de Vida. Doctora con mención internacional en Salud. Tutora de un programa de posgrado en Latinoamérica. Las principales investigaciones guardan relación con la línea de investigación: calidad de vida y factores psicosociales, y educación física y salud. ORCID ID: 0000-0003-2332-5601. Email: mlinares@ugr.es

\section{Tamara Espejo}

Máster Universitario en Investigación e Innovación en Currículum y Formación de la Universidad de Granada. Grado en Maestro de Educación Primaria en la Universidad de Granada. Ha colaborado en el proyecto "Programa de intervención educativa utilizando videojuegos activos y juegos motores para el apoyo a la docencia en materias de educación física y salud nutricional en los grados de primaria e infantil" (Referencia PID 14-81 de la Universidad de Granada). Ha disfrutado de una beca de colaboración en el departamento de Didáctica de la Expresión Musical, Plástica y Corporal de la 
Universidad de Granada. Ministerio de Educación y Ciencia. Investigadora en el grupo de investigación HUM-653 de la Universidad de Jaén. ORCID ID: 0000-0003-03637464. Email: tamaraeg@ugr.es

\section{Pilar Puertas}

Máster en Estudios Superiores de Lengua España, actualmente me encuentro realizando la tesis doctoral en el departamento de expresión musical, plástica y corporal y formo parte del grupo de investigación HUM-238. Graduada en Educación Primaria con mención en Educación Física. He asistido y participado en el I y II Congreso Internacional de Investigación y Didáctica de la Educación Física (Granada), en el I Congreso Internacional de Inteligencia Emocional y Bienestar (Zaragoza) y XXIV Congreso Internacional INFAD y en el I Congreso de Salud y Ciclo Vital (Almería). Participación como comité organizador y científico en el I y II Congreso Internacional de Investigación y Didáctica de la Educación Física, así como la publicación de artículos científico mayoritariamente relacionados con factores psicosociales dentro del ámbito de la educación y la actividad física y el deporte. ORCID ID: 0000-0001-8878-7677. Email: pilarpuertas@correo.ugr.es

\section{Ramón Chacón}

Máster Universitario en Investigación e Innovación en Currículum y Formación de la Universidad de Granada. Graduado en Educación Primaria con Mención en Educación Física. Desempeña su labor docente como Profesor Sustituto Interino en el departamento de Didáctica de la Expresión Corporal de la Universidad de Huelva. Pertenece al grupo de investigación "PROMECO" (Hum-238) desde diciembre de 2014, a través del cual ha desarrollado múltiples tareas de investigación, innovación y transferencia. Posee sesenta y dos publicaciones en revistas científicas, muchas de ellas indexadas en Web of Science y Scopus, dentro de las cuales es primer autor en más de veinte artículos. Del mismo modo, posee más de una veintena de capítulos de libro. Ha participado en más de una veintena de eventos de divulgación científica, considerando congresos internacionales y jornadas de investigación, mediante comunicaciones, ponencias y simposios. ORCID ID: 0000-0003-0937-1089. Email: rchacon@ugr.es 